\title{
Development of Liver Injury Simulation Model Using MPS Method
}

\author{
Yoshiki Hirahara \\ Graduate School of Engineering and Science \\ Shibaura Institute of Technology \\ 3-7-5, Toyosu Koutou-ku, Tokyo, Japan
}

\author{
Ken-ichi Tsubota \\ Department of Mechanical Engineering \\ Chiba University \\ 1-33, Yayoityo Inage, Chiba, Japan
}

\author{
Sota Yamamoto* \\ Department of Engineering Science and Engineering \\ Shibaura Institute of Technology \\ 3-7-5, Toyosu Koutou-ku, Tokyo, Japan \\ *Corresponding author
}

\begin{abstract}
The purpose of this work is developing the injury analysis model that can be expressed liver damage state by the Moving Particle Semi-implicit (MPS) method. In this paper, we set the material properties of the liver by simulating material test using the simplified geometric model that can represent the basic physical phenomena of the viscoelastic body.
\end{abstract}

Keywords-component; MPS method; liver; simultion; injruy

\section{INTRODUCTION}

In car accidents, abdominal organ injuries are frequently caused by seat belts which induce compression to the abdomen. Especially liver injury has a risk of massive bleeding and could be a cause of death.. Therefore much attention has been paid to reducing the injuries caused by the protection device. Accordingly, there is a need for injury assessment tool abdominal organs.

Recently finite element method is one of the major biomechanical analyzing methods. One of the shorts of FEM is description of collapsing and failure of the grids however it has great advantage in analyses of large deformation which are typical problems in the field of injury biomechanics. To describe the rupture and bleeding organs, we consider particle method. Particle method is one of mesh-less analyses, which is superior to large deformation and fluid analysis, and is expected to apply to biological field.

\section{METHOD}

Liver model is based on a three-dimensional red blood cell program by MPS method was provided by Tsubota et al [1] The structure of red blood cell has an analogy with the liver in that it is covered with a membrane containing an incompressible material. For this reason, it is considered that it is possible to construct the injury analysis model of the liver by applying this program.

To represents the viscoelastic behavior of liver tissue, we adopted Voigt elements between every neighboring particles. Based on this concept, we modified the Navier-Stokes equations as follows,

$$
\frac{D \mathbf{u}}{D t}=-\frac{1}{\rho} \nabla P+\nu \nabla^{2} \mathbf{u}+\frac{1}{\rho} \nabla \boldsymbol{\sigma}
$$

in which each variables denotes as follows, $\mathbf{u}$; the velocity vector, $\rho$; density, $P$; pressure, $v$; coefficient of viscosity, $\boldsymbol{\sigma}$; stress vector. The first term is the pressure section and the second term is the viscosity term of the Navier-Stokes equation, on the other hands the third term is the viscoelastic section of Voigt model. The stress vector of viscoelasticity term of Voigt model is represented as follows,

$$
\begin{gathered}
\boldsymbol{\sigma}_{i}=E \varepsilon_{i}+\eta_{i} \dot{\varepsilon} \\
\eta_{i}=A_{1} \varepsilon_{i}^{2}+A_{2} \varepsilon_{i}^{5}
\end{gathered}
$$

( $E$; elastic modulus, $\varepsilon$; strain, $\eta$; coefficient of viscosity, $\dot{\varepsilon}$; strain rate, $A_{1}, A_{2}$ : material constants). As shown in the equation (3), viscosity coefficient is a polynomial function of strain to expresses the nonlinearity of the strain rate dependency of the mechanical properties of liver.

Representation of fracture can be represented by eliminating the interaction between the particles when it exceeds a certain set value the strain. By storing the disconnected particles, the interaction in tension and shear between fracture surfaces would not be considered however the pressure is allowed to act in particles to each other through the fracture surfaces to determine the contact.

In the pressure term, negative pressure is dealt as well as positive pressure. It makes possible to express the Poisson effect in axial loading as controlling volumetric changes of material. Generally in MPS method, the initial particle number density is applied to the entire model which is defined at a 
well-inside part of the model. This definition causes negative pressure at particles on and nearby the surface in the initial state. To avoid this artifact, we defined the particle number density for each particle. The pressure of the liver parenchyma particles is set at 0 instead of determination of the free surface in fluid analysis by MPS method. Considering the compressibility, the compression ratio $\alpha$ is introduced to the Poisson equation of the pressure.

$$
\nabla^{2} P^{k+1}=-\frac{\rho_{0}}{\Delta t^{2}}\left(\frac{n-n^{0}}{n^{0}}-\alpha P_{i}^{k+1}\right)
$$

( $n$ :Particle number density, $n^{0}$ :Particle number density of the initial state, $\alpha$ :Compression ratio )

Using this program we conducted axial loading simulation for liver with simple geometry and evaluate the nominal stressstrain relationship and Poisson's ratio in comparison with experimental data from literature [2] and [3].

\section{RESUlTS}

We conducted a simulation of quasi-static tensile test of liver membrane and liver parenchyma (Fig1). The upper end of the specimen is restrained and the lower end is moved downward with a constant speed. Stress is calculated from the reaction force on the lower end, and the strain is defined as the nominal strain of the center part of the specimen. Liver membrane: breaking stress is $1.85 \mathrm{MPa}$ and breaking strain is $32.8 \%$. Liver parenchyma: breaking stress is $0.12 \mathrm{MPa}$ and breaking strain is $28.8 \%$. We have set of elastic modulus to fit to these values.

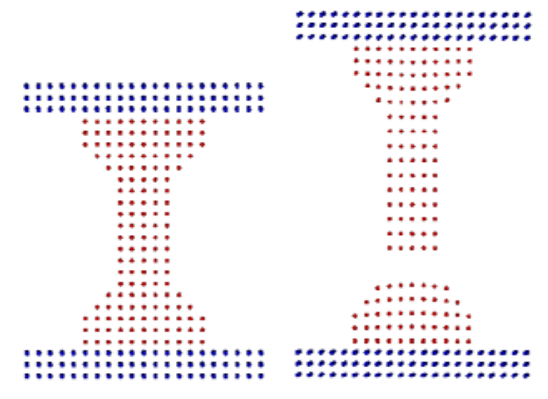

FIGURE I TENSIL TEST

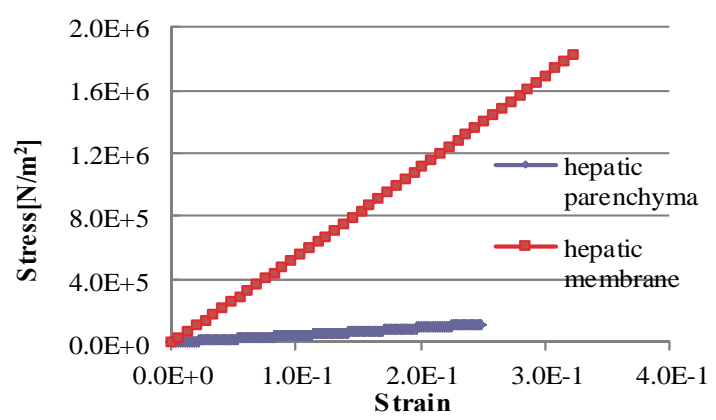

FIGURE II TENSILE STRESS STRAIN CURVE OF HEPATIC MEMBRANE AND PARENCHYMA
Next we conducted a simulation of the compression tests of liver parenchyma using a cubic model (Fig.3) and examine the relationship between the compression ratio in equation (4) and the nominal Poisson ratio of the liver model. Transversal strain, which is used to estimate the Poisson's ration, is calculated as the ration between the change and the initial of the width of the specimen. As a result, logarithmic graph of the compression ratio and Poisson's ratio shows an S-curve. As the compression ratio is close to 0 , Poisson's ration is asymptotic to 0.5 while it is getting to close to 0.2 with increasing the compression ratio (Fig.4). Poisson's ratio of pig liver is 0.4999 [3], which corresponds to the compression ratio is $3 \times 10^{-5}$ in our simulation.
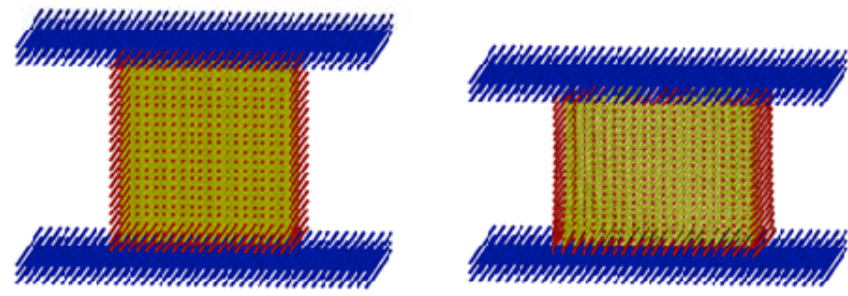

FIGURE III COMPRESSION TEST OF CUBE MODEL

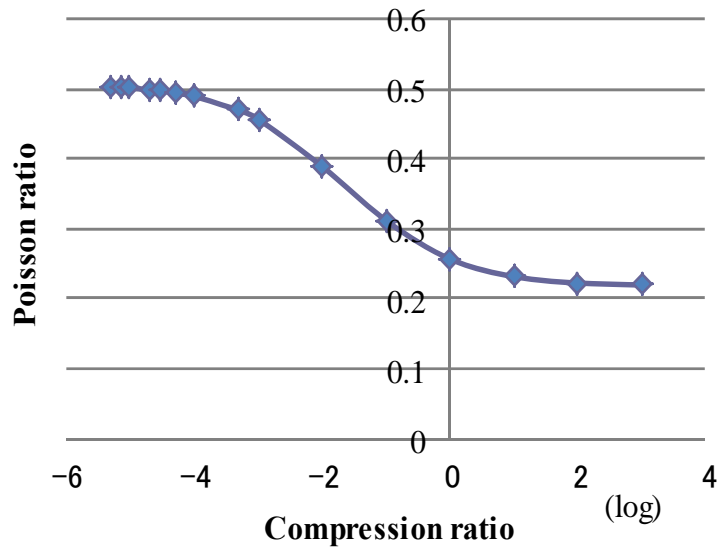

FIGURE IV RELATION OF POISSON RATIO AND COMPRESSION RATIO

\section{DISCUSSION}

Viscoelastic model by particle method was able to express the Poisson's ratio, breaking strain and breaking stress of the liver. These simulations are performed in a quasi-static rate, and so hardly leave the influence of viscosity. Therefore this model is necessary to adjust the viscosity term is for dynamic simulation to injury analysis.

\section{ACKNOWLEDGMENT}

I would like to give heartfelt thanks to associate professor Sota Yamamoto who offered continuing support and constant encouragement. Special thanks also go to professor Ken-ichi Tsubota whose comments made enormous contribution to my work. 


\section{REFERENCES}

[1] Ken-ichi Tsubota, Shigeo Wada, Effect of the natural state of an elastic cellular membrane on tank-treading and tumbling motions of a single red blood cell, PHYCICAL REVIEW E 81, 011910, 2010, 1-10

[2] A. Brunon, K. Bruyere-Garner, M. Cret, Mechanical characterization of liver capsule through uniaxial quasi-static tensile tests until failure, 2010, Journal of Biomechanics 43, 2221-2227

[3] Toshikazu Ishihara, Yuko Nakahira, Kazunori Furukawa, Measurement of the Mechanical Properties of the Pig Liver and Spleen, 2010, The Japan Society of Mechanical Engineers, 209-210 